\title{
Swarm behavior of self-propelled rods and swimming flagella
}

\author{
Yingzi Yang, ${ }^{1}$ Vincent Marceau, ${ }^{1,2}$ and Gerhard Gompper ${ }^{1}$ \\ ${ }^{1}$ Theoretical Soft Matter and Biophysics, Institut für Festkörperforschung, Forschungszentrum Jülich, D-52425 Jülich, Germany \\ ${ }^{2}$ Département de Physique, de Génie Physique et d'Optique, Université Laval, Québec, Québec, Canada G1V OA6
}

(Received 11 February 2010; revised manuscript received 17 August 2010; published 15 September 2010)

\begin{abstract}
Systems of self-propelled particles are known for their tendency to aggregate and to display swarm behavior. We investigate two model systems: self-propelled rods interacting via volume exclusion and sinusoidally beating flagella embedded in a fluid with hydrodynamic interactions. In the flagella system, beating frequencies are Gaussian distributed with a nonzero average. These systems are studied by Brownian-dynamics simulations and by mesoscale hydrodynamics simulations, respectively. The clustering behavior is analyzed as the particle density and the environmental or internal noise are varied. By distinguishing three types of cluster-size probability density functions, we obtain a phase diagram of different swarm behaviors. The properties of clusters such as their configuration, lifetime, and average size are analyzed. We find that the swarm behavior of the two systems, characterized by several effective power laws, is very similar. However, a more careful analysis reveals several differences. Clusters of self-propelled rods form due to partially blocked forward motion and are therefore typically wedge shaped. At higher rod density and low noise, a giant mobile cluster appears, in which most rods are mostly oriented toward the center. In contrast, flagella become hydrodynamically synchronized and attract each other; their clusters are therefore more elongated. Furthermore, the lifetime of flagella clusters decays more quickly with cluster size than of rod clusters.
\end{abstract}

DOI: 10.1103/PhysRevE.82.031904

PACS number(s): 87.17.Aa, 82.70.-y, 87.16.Qp

\section{INTRODUCTION}

Systems of self-propelled particles (SPPs), which exhibit an interaction mechanism that favors velocity alignment of neighboring particles, often display collective behaviors such as swarming and clustering. There are many examples for this swarming behavior, ranging from systems of microscopic particles (sperm, bacteria, and nanorods) to systems of macroscopic objects (birds and fish).

Since the pioneering simulation work of Vicsek et al. [1], SPP systems have attracted a lot of interest at the theoretical [2-8] and computational [9-15] levels. Typically, in simulation models of swarm behavior, pointlike agents move with an imposed nonzero velocity and tend to align their direction of motion with others in a prescribed neighborhood $[1,10,11,14]$. Although the alignment mechanism may differ from one model to the other, the basic properties of swarm behavior are quite universal [16]. Upon variation of parameters such as particle density, particle velocity, or environmental noise, the system can undergo a transition from a disordered state, where the average total velocity or orientation vanishes, to a nematically ordered state. Near the transition point, the cluster-size probability density function (PDF) is characterized by a power-law decay $[11,16]$. For intermediate densities, phase separation into regions of different densities and band formations has been found [15].

Self-propelled motion is common in biological systems at microscopic or mesoscopic length scales, such as suspensions of bacteria, like E. coli [17] and Bacillus subtilis [18-20], or tissue cells (keratocytes) [9], whose sizes are all on the micrometer scale. A special class of biological systems are rodlike self-propelled particles (rSPP), for example, myxobacteria (approximately $10 \mu \mathrm{m}$ long) [21,22]. When starved, myxobacteria are elongated to an average aspect ratio of approximately $1: 7$, glide on a substrate along their long axes and undergo a process of alignment, rippling, streaming, and aggregation that culminates in a three-dimensional fruiting body. A model, which takes into account the exchange of a morphogen during cell-cell contact and a preferred cell motion in the direction of largest morphogen concentration, has been designed to describe the streaming and two-stage aggregation of myxobacteria [23].

Sperm (with a length of about $50 \mu \mathrm{m}$ ) [24,25] and nematodes [26] (about $1 \mathrm{~mm}$ long) employ a sinusoidal undulation of their slender bodies to push the fluid backward and to propel themselves forward. Large trainlike clusters of wood mouse sperm $[27,28]$ are believed to result in greater thrust forces to move more efficiently through a highly viscous environment. The wood mouse sperm has a hooklike structure at its head, by which it can be hitched to the midpart or the tail of a neighboring cell for robust cooperation. However, nematodes which do not have hook structures also display a pronounced tendency to adhere to each other in a film of water, to form assemblies consisting of many organisms, and to exhibit a striking coordinated movement [26]. Also, sea urchin sperm organize into a hexagonal pattern of rotating vortices at surfaces [29].

A nice physical realization of self-propelled rods (SPRs) are bimetallic nanorods consisting of long Pt and Au segments [30]. The rods, about $300 \mathrm{~nm}$ in diameter and $2 \mu \mathrm{m}$ long, move autonomously in an aqueous hydrogen peroxide solutions by catalyzing the formation of oxygen at the $\mathrm{Pt}$ end. They move predominantly in the direction of the Pt end, with a velocity depending on the concentration of hydrogen peroxide. When a gradient of the hydrogen peroxide concentration is imposed, the rods exhibit directed motion toward regions of higher concentrations through active diffusion [31].

A related system is a fluidized monolayer of macroscopic rods in the nematic liquid-crystalline phase [32]. The rods confined between two hard walls are energized by an exter- 
nal vertical vibration and gain kinetic energy through frequent collisions with the floor and the ceiling of the container. Long-lived giant number fluctuations are found, which shows that simple contact can give rise to flocking, coherent swirling motion, and large-scale inhomogeneities [33]. However, in this experiment, the rods do not have a preferred direction of motion.

All of these examples of self-propelled particles employ different propulsion mechanisms and have different interactions. However, their swarm behavior, such as flocking, streaming, and clustering, is surprisingly similar. The common characteristic of these systems is their rodlike structures and their quasi-two-dimensional active motion. Myxobacteria glide on surfaces [21], while sperm and nematodes gather at substrates $[26,29,34]$. In suspensions of rodlike particles in thermal equilibrium, volume exclusion favors the alignment of rods. At high densities, it stabilizes a nematic state characterized by long-range orientational order [35].

While constant-velocity polar point particles interacting locally by nematic alignment in the presence of noise have been studied intensively in recent years [1-15], much less is known theoretically about the behavior of elongated particles with volume exclusion or about the collective behavior of swimmers with hydrodynamic interactions. Previous simulation studies of SPRs in two dimensions show that selfpropelled motion enhances the tendency for nematic ordering [36], as well as aggregation and clustering [37]. Also, rods have an increased probability to be located near surfaces (depending on their velocity, length, and thermal noise) [38] and form hedgehoglike clusters at surfaces [39]. In Ref. [37], two regimes of clustering have been distinguished by their unimodal or bimodal weighted cluster-size distribution functions; however, the system contained a relatively small number of particles compared to those employed in simulation studies of swarming of SPPs. Continuum equations for the description of SPR systems have been derived recently within a mean-field approximation $[6,7]$. This theory predicts that hard-core interactions are insufficient to generate a macroscopically polarized state, because they cannot distinguish the two ends of a rod, and makes interesting predictions for the fluctuations in the nematic and isotropic states (such as a crossover from diffusive to propagating density fluctuations). However, the mean-field approximation of volume exclusion has the limitation of omitting correlation effects, and thus works best for slowly varying density distributions.

In addition, hydrodynamic interactions between rSPPs have so far been largely neglected. These interactions depend on the type of self-propulsion, where "pullers" repel and "pushers" attract each other [40,41]. Nematic suspensions of swimming rodlike pushers are found to be unstable at long wavelengths as a result of hydrodynamic fluctuations [42]. For sperm and flagella, it has been shown theoretically that the hydrodynamic coupling synchronizes the phases of their sinusoidal beating tails $[24,43,44]$. Also, the hydrodynamic interaction between these microswimmers implies attraction and cluster formation [43]; similarly, it makes an essential contribution to the capturing of sperm near walls [45]. However, the relative importance of directed self-propulsion, particle shape, volume exclusion, and hydrodynamic interactions to the emergence of swarm behavior remains unclear.
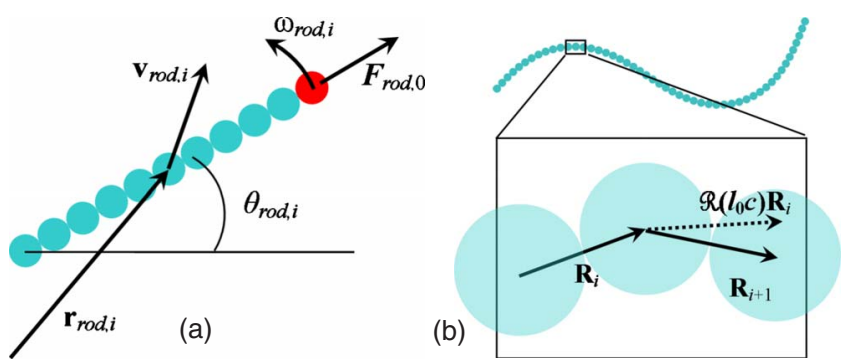

FIG. 1. (Color online) (a) Model of a self-propelled rod and the coordinates used in two dimensions. The rod is discretized into $n_{\text {rod }}=L_{\text {rod }} / l_{b}$ beads for the calculation of the volume-exclusion interaction. (b) Model of a flagellum in two dimensions.

In this paper, we employ a model of hard rods with strict volume exclusions and simulate large systems containing at least 1000 particles. We focus on rSPP systems at a density below the isotropic-nematic transition of Brownian rods. We employ a model consisting of rigid SPR performing an overdamped translational motion in two dimensions and analyze the resulting cluster-size probability density distribution, cluster configurations, and lifetimes. Three types of clustersize probability density distribution functions allow us to distinguish three different states and to construct a phase diagram as a function of particle density and environmental noise. As a special case of rSPP with an explicit propulsion mechanism, we investigate a suspension of flagella, which move by sinusoidal beating of their bodies in a twodimensional fluid. The motion of the surrounding fluid is described by particle-based mesoscopic simulation method called multiparticle collision dynamics (MPC) $[46,47]$. This method has been shown to capture the full hydrodynamics and flow behavior of complex fluids over a wide range of Reynolds numbers very well [48]. By comparing the results for SPR and flagella, we elucidate the contribution of hydrodynamic interactions to the swarm behavior.

This paper is organized as follows. Section II gives a brief description of our models and simulation methods. We analyze the collective behavior of SPR systems in Sec. III. In Sec. IV, we study the swarm behavior of flagella and compare the results obtained with both models. The influence of hydrodynamic interactions and the flagellar beat on the swarm behavior is discussed. We summarize our main conclusions in Sec. V.

\section{MODELS AND SIMULATION TECHNIQUES}

\section{A. Self-propelled rods}

We consider a system of $N_{\text {rod }}$ rods of length $L_{\text {rod }}$ in a two-dimensional simulation box of size $L_{x} \times L_{y}$. Each rod is characterized by an orientation angle $\theta_{\text {rod,i }}$ with respect to the $x$ axis, a center-of-mass position $\mathbf{r}_{r o d, i}$, a center-of-mass velocity $\mathbf{v}_{\text {rod,i }}$, and an angular velocity $\omega_{\text {rod,i }}$ around its center of mass [see Fig. 1(a)]. The rods move ballistically according to their velocities,

$$
\mathbf{r}_{r o d, i}\left(t+\Delta t_{\text {rod }}\right)=\mathbf{r}_{\text {rod }, i}(t)+\mathbf{v}_{\text {rod }, i}(t) \Delta t_{\text {rod }},
$$




$$
\theta_{\text {rod }, i}\left(t+\Delta t_{\text {rod }}\right)=\theta_{\text {rod }, i}(t)+\omega_{\text {rod }, i}(t) \Delta t_{\text {rod }},
$$

where $\Delta t_{\text {rod }}$ is the simulation time step. The particle velocity can be decomposed into a parallel and a perpendicular component relative to the rod axis, $\mathbf{v}_{\text {rod, } i}=\mathbf{v}_{\text {rod, } i, \|}+\mathbf{v}_{\text {rod,i, }, \perp}$.

We consider the rods to be embedded in an overdamped fluid medium where hydrodynamics can be approximated by an anisotropic friction on the rodlike particles. The motion is then determined by

$$
\begin{aligned}
& \mathbf{v}_{\text {rod }, i, \|}(t)=\frac{1}{\gamma_{\|}}\left(\sum_{j \neq i}^{N_{\text {rod }}} \mathbf{F}_{i j, \|}+\xi_{\|} \mathbf{e}_{\|}+F_{r o d, 0} \mathbf{e}_{\|}\right), \\
& \mathbf{v}_{r o d, i, \perp}(t)=\frac{1}{\gamma_{\perp}}\left(\sum_{j \neq i}^{N_{\text {rod }}} \mathbf{F}_{i j, \perp}+\xi_{\perp} \mathbf{e}_{\perp}\right), \\
& \omega_{\text {rod }, i}(t)=\frac{1}{\gamma_{r}}\left(\sum_{j \neq i}^{N_{\text {rod }}} M_{i j}+\xi_{r}\right),
\end{aligned}
$$

where $\mathbf{e}_{\|}$and $\mathbf{e}_{\perp}$ are the local parallel and perpendicular unit vectors of the rod orientation. $F_{\text {rod }, 0}$ is a constant propelling force applied along $\mathbf{e}_{\|}$. The friction coefficients are given by $\gamma_{\perp}=2 \gamma_{\|}, \gamma_{\|}=L_{\text {rod }}$, and $\gamma_{r}=\gamma_{\|} L_{r o d}^{2} / 6$. The random forces $\xi_{\|}$, $\xi_{\perp}$, and $\xi_{r}$ are white noises, which are determined by their variances $\sigma_{\text {rod }}^{2} L_{\text {rod }}, \sigma_{\text {rod }}^{2} L_{\text {rod }}$, and $\sigma_{\text {rod }}^{2} L_{\text {rod }}^{3} / 12$, respectively. Finally, $\mathbf{F}_{i j}$ is the force generated by volume exclusion between rods $i$ and $j$, and $M_{i j}$ is the torque generated by $\mathbf{F}_{i j}$ on $\operatorname{rod} i$ in the reference system of center of mass of $\operatorname{rod} i$.

For the calculation of the interactions, each rod is discretized into $n_{\text {rod }}=L_{\text {rod }} / l_{b}$ beads of diameter $l_{b}$, as illustrated in Fig. 1(a). The volume exclusion between rods is then modeled by a shifted and truncated Lennard-Jones potential,

$$
V(r)= \begin{cases}4 \epsilon\left[\left(\frac{l_{b}}{r}\right)^{12}-\left(\frac{l_{b}}{r}\right)^{6}\right]+\epsilon, & r<2^{1 / 6} l_{b} \\ 0, & r \geq 2^{1 / 6} l_{b},\end{cases}
$$

between beads belonging to different rods, where $r$ is the distance between two beads, $l_{b}$ is the bead diameter, and $\epsilon$ is the strength of the potential. We use $\epsilon$ as the energy scale in our SPR simulations.

A single rod without noise then moves with a constant velocity $v_{0}=F_{\text {rod }, 0} / \gamma_{\|}$. In the nonzero noise regime, the diffusion constant along the parallel direction is $D_{\|}$ $=\sigma_{\text {rod }}^{2} L_{\text {rod }} \Delta t_{\text {rod }} / 2 \gamma_{\|}^{2}$. The dimensionless Péclet number, which measures the ratio of self-propelled and diffusive motions, is thus

$$
\mathrm{Pe}=\frac{L_{r o d} v_{0}}{D_{\|}}=\frac{2 \gamma_{\|} F_{r o d, 0}}{\sigma_{\text {rod }}^{2} \Delta t_{\text {rod }}} .
$$

We use $1 / \mathrm{Pe} \propto \sigma_{\text {rod }}^{2}$ to characterize the strength of the environmental noise.

In SPR systems [6,37], alignment is naturally introduced by the volume exclusion between the anisotropic particles; this also implies that the interaction neighborhood needs no further assumptions but is directly related to the rod length. Hard-core interactions do not distinguish the two ends of an symmetrically elongated object. Thus, both parallel and an- tiparallel velocity configurations are induced. In simulations of pointlike SPPs, noise is implemented by adding a random component to the velocity orientation of each particle. In our model of SPR, random forces are applied on each rod, which results in fluctuations in both the magnitude and the orientation of the velocity vectors. For a single rod, the orientation fluctuations lead to rotational diffusion, which implies a persistence length

$$
L_{p}=\frac{2 v_{0} \gamma_{\|}^{2}}{\sigma_{r o d}^{2} L_{r o d} \Delta t}
$$

of its trajectory. Note that the noise forces are not caused by thermal fluctuations, which would require a factor of 2 between the variance of the random forces in parallel and perpendicular directions. In most biological and synthetic rSPP systems, thermal fluctuations are indeed negligible due to large sizes of the particles. In these systems, the environmental noise arises, for example, from density fluctuations of signaling molecules for chemotactic swimmers or from fluctuations of the motor activity.

We use rods of length $L_{\text {rod }}=11 l_{b}$ and undisturbed velocity $v_{0}=1.21 \epsilon /\left(\gamma L_{\text {rod }}\right)$. Effects of a polydispersity of rod lengths or a distribution of propulsion forces are not considered. The motion of rods is calculated with a discrete time step $\Delta t_{\text {rod }}$ $=0.001$. Most of our rod simulations start from random initial states, where the rods are placed into the simulation box with random orientations and random positions without overlap. If not explicitly mentioned, the size of the simulation box is $L_{x}=L_{y}=400 l_{b}$, which is much larger than the rod length. Periodic boundary conditions are employed.

Our model differs from the model of Ref. [37] by the type of repulsive interaction between the rods. In Ref. [37], rods interact by a "soft" volume exclusion, where the repulsion force is proportional to the square of overlapping area, while in our model the interaction is a short-range Lennard-Jones potential between discretized beads. In the limit of a large overlap energy, the two models become equivalent.

\section{B. Flagella}

We consider a system of $N_{f l}$ flagella of length $L_{f l}$ in a box of size $L_{x} \times L_{y}$. Each flagellum consists of semiflexible string of monomers of mass $m_{f l}$, connected by springs [see Fig. $1(b)]$. The shape of the flagellum is determined by the elastic energy

$$
E=\sum_{i} \frac{k}{2 l_{0}^{2}}\left(\left|\mathbf{R}_{i}\right|-l_{0}\right)^{2}+\sum_{i} \frac{\kappa}{2 l_{0}^{3}}\left\{\mathbf{R}_{i+1}-\mathfrak{R}\left(l_{0} c\right) \mathbf{R}_{i}\right\}^{2}+V .
$$

Here, the first term is the harmonic potential generated by springs with spring constant $k$ and rest length $l_{0} . \mathbf{R}_{i}$ is the bond vector pointing from monomer $i$ to monomer $(i+1)$. The second term of Eq. (9) is the bending energy of the flagellum, with bending rigidity $\kappa . \mathfrak{R}\left(l_{0} c\right)$ is an operator which rotates a two-dimensional vector clockwise by an angle $l_{0} c$. The local spontaneous curvature $c$ varies with time $t$ and position $x$ along the flagellum to generate a propagating bending wave, 


$$
c(x, t)=A \sin [-2 \pi f t+q x+\varphi] .
$$

The detailed analysis of the beating pattern of nematodes [26] and bull sperm $[25,49]$ has shown that a single sine mode represents the beating pattern to a good approximation. We use the wave number $q=2 \pi / L_{f l}$, such that the phase difference between the first and the last monomers is $2 \pi$, and one complete wavelength is present on the flagellum. The beating frequency $f$ is constant for each flagellum; it is chosen from a Gaussian distribution, centered at $f_{0}$ and with variance $\sigma_{f l}^{2} f_{0}^{2} . \varphi$ is the initial phase of the first monomer, which is chosen from a uniform distribution in $[0,2 \pi]$. As $t$ increases, a wave propagates along the flagellum from the first to the last monomer, pushing the fluid backward and propelling the flagellum forward. Although the spontaneous local curvature $c$ is prescribed by Eq. (10), the flagellum is elastic and its configuration is affected by the viscosity of the medium and the flow field generated by other flagella. The third term in Eq. (9) describes the interaction between flagella due to volume exclusion; here, we employ again the shifted and truncated Lennard-Jones potential [Eq. (6)] between monomers of different flagella.

Our model of a flagellum differs from the model of a sperm employed in Ref. [43] by the absence of a passive midpiece and a circular head. Also, in the sperm simulations [43], two sine waves were present on the tail, while a single sine wave is present on the flagellum.

We use flagella of length $L_{f l}=50 l_{0}$. The elastic moduli in Eq. (9) are the spring constant $k=1.25 \times 10^{4} k_{B} T$ and the bending rigidity $\kappa=200 k_{B} T L_{f l}$. The amplitude $A=5 / L_{f l}$ of the spontaneous curvature in Eq. (10) induces a beating amplitude of about $6.1 l_{0}=0.12 L_{f l}$. The strength $\epsilon=15 k_{B} T$ of the volume exclusion is large compared to the thermal energy. The simulations are initialized by placing $N_{f l}$ flagella in the simulation box with random initial positions and orientations, without any overlap. The size of the simulation box is $L_{x} \times L_{y}$, where $L_{x}=L_{y}=400 l_{0}$, eight times the length of a flagellum. Periodic boundary conditions are employed.

Each simulation run of the flagella systems covers a total time interval of about 3300 beats. The first 800 beats are not taken into account in the calculation of averages, in order to allow the system to reach the stationary state. This time is longer than the largest relaxation time of about 650 beats observed in the system with a width $\sigma_{f l}=0.1 \%$ of the frequency distribution.

\section{MPC}

MPC is a particle-based mesoscopic simulation technique used to describe the hydrodynamics and flow behavior of complex fluids. The fluid is modeled by $N_{s o l}$ point particles of mass $m_{s o l, i}$, which are characterized by their continuous space position $\mathbf{r}_{s o l, i}$ and velocity $\mathbf{v}_{s o l, i}$. During every time step $\Delta t_{M P C}$, there are two distinct simulation steps: streaming and collision. In the streaming step, the fluid particles do not interact with each other and move ballistically according to their velocities,

$$
\mathbf{r}_{s o l, i}\left(t+\Delta t_{M P C}\right)=\mathbf{r}_{s o l, i}(t)+\mathbf{v}_{s o l, i}(t) \Delta t_{M P C} .
$$

In the collision step, the particles are sorted into the cells of a square lattice of side length $a$ according to their position and interact with all other particles in same collision box through a multibody collision. The collision step is defined by a rotation of all particle velocities in a box in a comoving frame with its center of mass. Thus, the velocity of the $i$ th particle in the $j$ th box after collision is

$$
\mathbf{v}_{s o l, i}\left(t+\Delta t_{M P C}\right)=\mathbf{v}_{c m, j}(t)+\Re_{j}(\alpha)\left[\mathbf{v}_{s o l, i}-\mathbf{v}_{c m, j}\right],
$$

where

$$
\mathbf{v}_{c m, j}(t)=\frac{\sum_{j} m_{s o l, i} \mathbf{v}_{s o l, i}}{\sum_{j} m_{s o l, i}}
$$

is the center-of-mass velocity of the $j$ th box and $\Re_{j}(\alpha)$ is a rotation matrix which rotates a vector by an angle $\pm \alpha$, with the sign chosen at random. This implies that during the collisions particles exchange momentum, but the total momentum and kinetic energy are conserved within each collision box. In order to ensure Galilean invariance, a random shift of the collision grid has to be performed [50].

The total kinematic viscosity $\nu$ is the sum of two contributions: the kinetic viscosity $\nu_{k i n}$ and the collision viscosity $\nu_{\text {coll }}$. In two dimensions, approximate analytical expressions are $[51,52]$

$$
\begin{aligned}
& \frac{\nu_{\text {coll }}}{\sqrt{k_{B} T a^{2} / m_{\text {sol }}}}=\frac{1}{12 h}(1-\cos \alpha)\left(1-\frac{1}{\rho}\right), \\
& \frac{\nu_{\text {kin }}}{\sqrt{k_{B} T a^{2} / m_{\text {sol }}}}=h\left[\frac{1}{1-\cos \alpha} \frac{\rho}{\rho-1}-\frac{1}{2}\right],
\end{aligned}
$$

where $\rho$ is the average particle number in each box and $h$ $=\Delta t_{M P C} \sqrt{k_{B} T / m_{\text {sol }} a^{2}}$ is the rescaled mean free path. We use $k_{B} T=1, m=1, a=1, \Delta t_{M P C}=0.025, \alpha=\pi / 2$, and $\rho=10$. This implies, in particular, that the simulation time unit $\left(m a^{2} / k_{B} T\right)^{1 / 2}$ equals unity. With these parameters, the total kinematic viscosity of fluid is $\nu=\nu_{\text {coll }}+\nu_{\text {kin }} \approx 3.02$.

During the MPC streaming step, the equations of motion of the flagella monomers are integrated using a velocityVerlet algorithm, with a molecular-dynamics time step $\Delta t_{f l}$ $=\Delta t_{M P C} / 50=5 \times 10^{-4}$. The bond length between the monomers is related to the collision cell size by $l_{0}=a / 2$. The flagella only interact with the fluid during the MPC collision step. This is done by sorting the flagella monomers together with the fluid particles into the collision cells and rotating their velocities relative to the center-of-mass velocity of each cell. Since energy is continuously injected into the system by the actively beating flagella, we employ a thermostat to keep the fluid temperature constant by rescaling all fluid-particle velocities in a collision box relative to its center-of-mass velocity after each collision step.

With the parameters given above, a single flagellum with $f_{0}=1 / 120$ swims forward with the velocity $v_{\text {single }}$ $=0.020 \pm 0.001$ in a MPC fluid. Thus, we estimate a Reynolds number $\mathrm{Re}=2 A_{f l} v_{\text {single }} / \nu \approx 0.04$ for our flagellum model, where $A_{f l}=0.12 L_{f l}$ is the beating amplitude. The velocity of our flagella can be compared with the velocity of an infinitely long string beating in a two-dimensional fluid at $\mathrm{Re}=0$, which was calculated analytically by Taylor [24] to be 
TABLE I. Definition of power-law exponents for the cluster-size distribution $\Pi(n)$, the average cluster size $\langle n\rangle$, and the cluster lifetime $T_{\text {life }}$, and their typical values for rods and flagella.

\begin{tabular}{lccc}
\hline \hline & Power law & Rods & Flagella \\
\hline$\Pi(n)$ & $n^{\beta}$ & $-6<\beta \simeq-2$ & $-4<\beta \simeq-2$ \\
$\langle n\rangle$ & $\mathrm{Pe}^{\zeta}$ & $\zeta=0.37$ & \\
$\langle n\rangle$ & $\sigma_{f l}^{-\zeta}$ & & $\zeta=0.26$ \\
$T_{\text {life }}$ & $n^{-\delta}$ & $\delta=0.2$ & $\delta=0.5$ \\
\hline \hline
\end{tabular}

$$
v_{\text {single }}=\frac{2 \pi A_{f l}^{2} v_{\text {wave }}}{\lambda_{\text {wave }}^{2}}\left(1-\frac{19}{4} \frac{\pi^{2} A_{f l}^{2}}{\lambda_{\text {wave }}^{2}}\right)
$$

where $\lambda_{\text {wave }}$ is the wavelength and $v_{\text {wave }}=\lambda_{\text {wave }} f$ is the propagation velocity of the sinusoidal wave on the flagellum. Applying the parameters in our simulations, we obtain $v_{\text {single }}=0.0183$, in excellent agreement with the simulation result. This demonstrates that the simulation model describes the limit of low-Reynolds-number hydrodynamics very well.

\section{SWARMING BEHAVIOR OF SELF-PROPELLED RODS}

After starting from a random initial state, the rods aggregate and form clusters. Large clusters can form by collisions of smaller ones, while at the same time they can break up due to collisions with other clusters or due to the noisy environment. After a transient phase, the system reaches a stationary state, in which the formation rate of any cluster size equals its breakup rate. The degree of aggregation in the system depends on its parameters such as the Péclet number and the number density $\rho_{\text {rod }}=N_{\text {rod }} / L_{x} L_{y}$.

We define a cluster as follows. We consider two rods to be in the same cluster if the angle between their orientation vectors is less than $\pi / 6$ and the nearest distance is less than $2 l_{b}$, which is about two times the width of a rod. A cluster is defined as a set of rods that are neighbors either directly or through other rods at a given moment in time. Its size is simply the number of rods it contains. A freely gliding rod without any neighbor is considered as a cluster of size $n=1$.

We study systems at intermediate densities, where $\rho_{\text {rod }}$ is neither very low, such that there are hardly any collisions, nor high enough for a nematic phase to appear for rods in thermal equilibrium, i.e., densities lower than the critical density $\rho_{c}=3 \pi /\left(2 L_{\text {rod }}^{2}\right)$ of the isotropic-to-nematic phase transition [35]. The statistical quantities, which will be analyzed in Secs. III and IV, are listed in Table I.

\section{A. Cluster-size probability density functions and stationary states}

For a system with particles distributed at random in space, the probability of finding $n$ particles in some area obeys a binomial distribution; in our SPR systems, the probability to find large particle numbers $n$ is increased by aggregation and clustering. The stationary cluster-size $\operatorname{PDF} \Pi(n)$ results from the balance between the cluster formation and breakup rates. While the former depends on the collision rate of clusters,
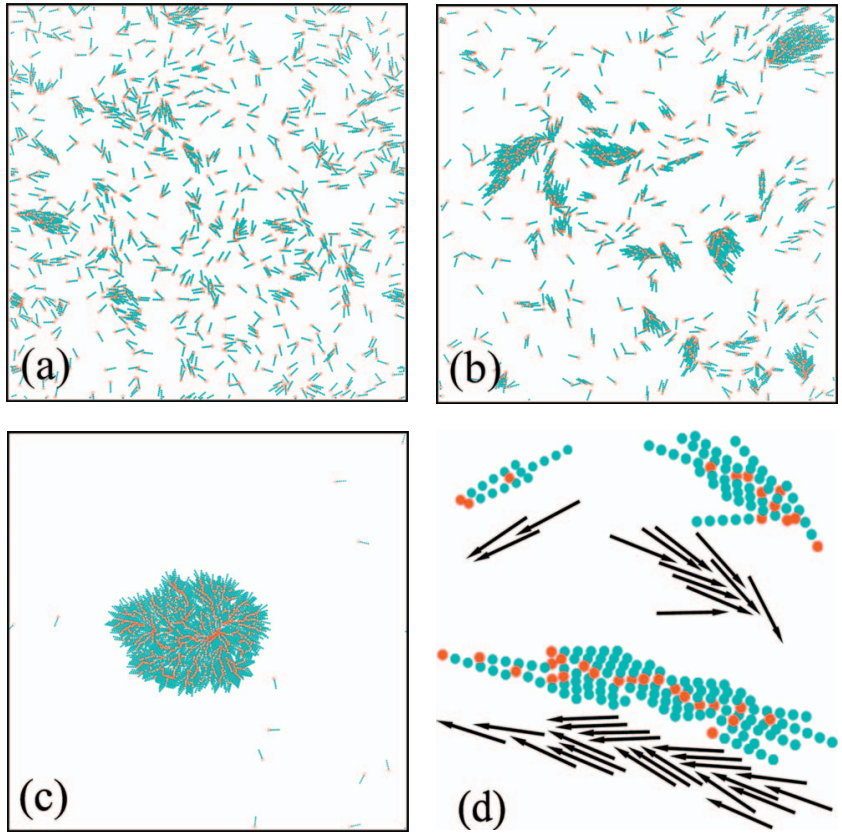

FIG. 2. (Color) Snapshots of the SPR systems at different stationary states. Parameters are $\rho_{\text {rod }} L_{\text {rod }}^{2}=0.7744$ and (a) $1 / \mathrm{Pe}$ $=0.02645\left(\Pi_{1}\right)$, (b) $1 / \mathrm{Pe}=0.00501\left(\Pi_{2}\right)$, and (c) $1 / \mathrm{Pe}=0.00041$ $\left(\Pi_{3}\right)$. Red dots mark the front ends of the rods. (d) Close-up of clusters of sizes $n=3,10$, and 22 shows the partially blocked structure; chosen from a simulation with parameters $\rho_{\text {rod }} L_{\text {rod }}^{2}=0.7744$ and $1 / \mathrm{Pe}=0.00095$. For a movie see Ref. [53]

the latter depends also on the environmental noise and the cluster size. We distinguish three different stationary states in our SPR systems by comparing the shapes of their corresponding PDFs. Snapshots are shown in Fig. 2 and a movie can be found in Ref. [53].

A disordered state, where rods are distributed in the whole space and oriented in different directions, is characterized by a PDF denoted as $\Pi_{1}$ in Fig. 3. In a snapshot [Fig. 2(a)], a weak aggregation tendency can be recognized in this case, where several small clusters of well polarized members glide in arbitrary directions. $\Pi_{1}$ decreases as a power law for small

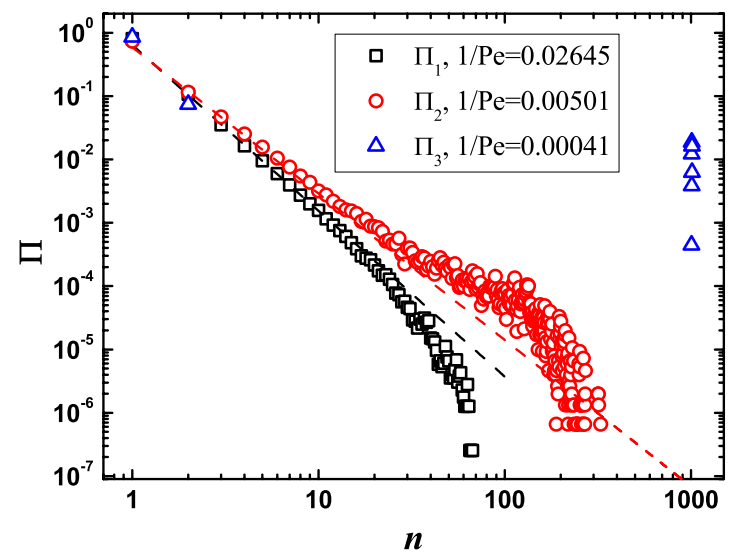

FIG. 3. (Color online) Cluster-size distribution functions $\Pi(n)$ for systems shown in the snapshots of Fig. 2(a), $\Pi_{1}$ ( $\square$, black); Fig. 2(b), $\Pi_{2}\left(\bigcirc\right.$, red); and Fig. $2(\mathrm{c}), \Pi_{3}(\triangle$, blue). 
cluster sizes, and then decays exponentially for large $n$. The same kind of PDF has also been found in simulations of swarms of pointlike SPP interacting via a phenomenological alignment mechanism $[11,16]$. The range of the power-lawdecay regime of $\Pi_{1}$ depends on the rod density and the environmental noise. Increasing density or decreasing noise shifts the exponential cutoff to larger $n$.

The system with the second type of PDF, denoted $\Pi_{2}$ in Fig. 3, is more ordered, with an obvious tendency to form large clusters. A snapshot [Fig. 2(b)] shows several large and motile clusters moving in different directions. $\Pi_{2}$ also displays a power-law decay at small cluster sizes, but shows an increased probability (compared to the power-law decay) of finding large clusters. Increasing the number density or decreasing the noise shifts the prominent shoulder to larger cluster sizes. For very large aggregates, greater than the shoulder location, $\Pi_{2}$ decreases rapidly.

The system with the third type of PDF, denoted $\Pi_{3}$ in Fig. 3 , is characterized by a giant cluster, in which most rods are oriented radially toward the center [Fig. 2(c)]. The giant cluster forms when several smaller motile clusters collide head on in a short time interval, such that a nucleus with a blocked structure emerges. This nucleus continues to grow until most of rods in the system are gathered in it. $\Pi_{3}$ has two parts: a peak at large $n$ representing the giant clusters and another peak at very small $n$ corresponding to some freely swimming rods not collected by the giant cluster. The average rod density outside the giant clusters is very low.

Both $\Pi_{1}$ and $\Pi_{2}$ display a power-law decay at small cluster sizes,

$$
\Pi \sim n^{\beta} .
$$

The exponent $\beta$ is a function of the rod density $\rho_{\text {rod }}$ and noise $1 / \mathrm{Pe}$; it increases with increasing $\rho_{\text {rod }}$ and decreases with increasing 1/Pe (Fig. 4). However, the dependence of $\beta$ on $\rho_{\text {rod }}$ or $1 / \mathrm{Pe}$ in the $\Pi_{1}$ regime is much stronger than in the $\Pi_{2}$ regime; in the latter case, $\beta$ approaches -2 .

By systematically varying the rod density $\rho_{\text {rod }}$ and the environmental noise level, we can construct a phase diagram with regions characterized by different types of PDFs (see Fig. 5). Clearly, $\Pi_{1}$ is found in the low-density and highnoise regime, $\Pi_{3}$ is found in the high-density and low-noise regime, and $\Pi_{2}$ is associated with the transition region between $\Pi_{1}$ and $\Pi_{3}$. Note that all systems in Fig. 5 were started from disordered initial states. Systems characterized by the probability density function $\Pi_{2}$ bear some similarity with liquid systems supercooled below the freezing point. Note that the system with $1 / \mathrm{Pe}=0.00095$ and $\rho_{\text {rod }} L_{\text {rod }}^{2}=0.7744$ in Fig. 5 displays both $\Pi_{2}$ and $\Pi_{3}$ distributions corresponding to simulations with different initial random states. Systems with the probability density function $\Pi_{3}$ show the characteristics of a glassy behavior, where the dense packing of rods arises from the random collisions and remains frozen at later times.

Our results are consistent with those of Ref. [37]. By comparing short runs for systems with and without fluctuations, the transition from $\Pi_{1}$ to $\Pi_{2}$ was found in Ref. [37] to shift to larger values of the aspect ratio $L_{\text {rod }} / l_{b}$ and total area
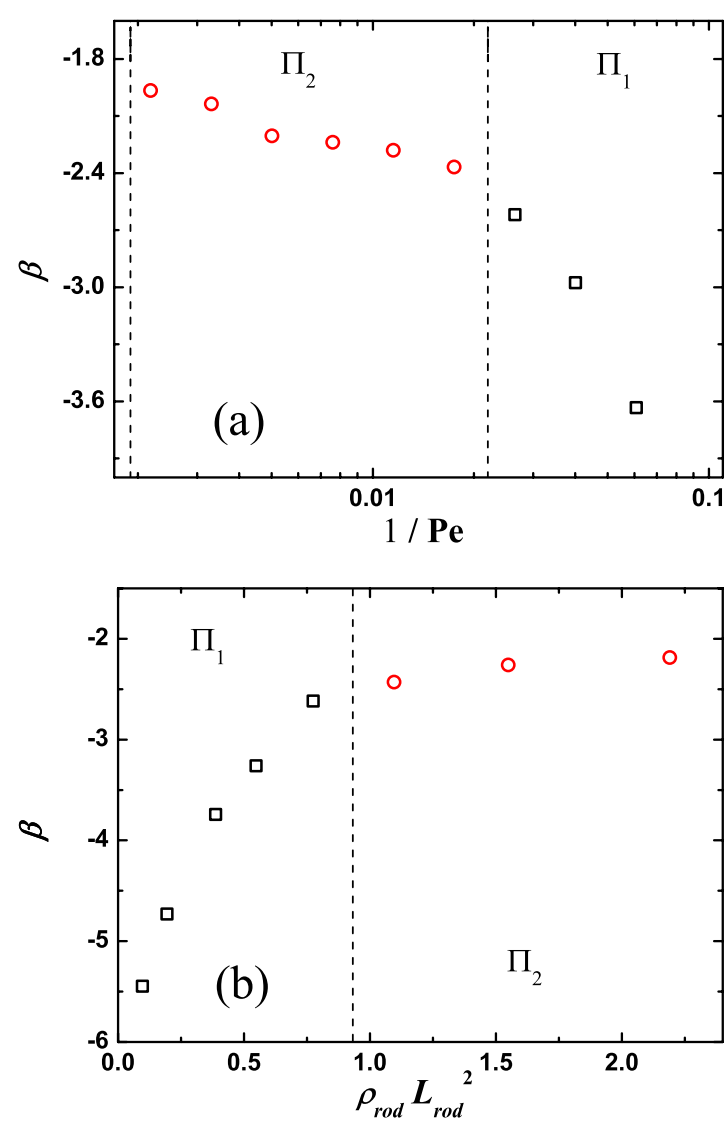

FIG. 4. (Color online) The exponent $\beta$ of the power-law part of $\Pi_{1}$ and $\Pi_{2}$ as a function of (a) the environmental noise $1 / \mathrm{Pe}$ when $\rho_{\text {rod }} L_{\text {rod }}^{2}=0.7744$ and (b) the rod density $\rho_{\text {rod }} L_{\text {rod }}^{2}$ when $1 / \mathrm{Pe}$ $=0.02645$.

fraction of rods $\eta=\rho_{\text {rod }} L_{\text {rod }} l_{b}$. Figure 5 demonstrates that in our system the transition shifts with increasing $1 /$ Pe to larger $\rho_{\text {rod }} L_{\text {rod }}^{2}$, which is proportional to $\eta L_{\text {rod }} / l_{b}$.

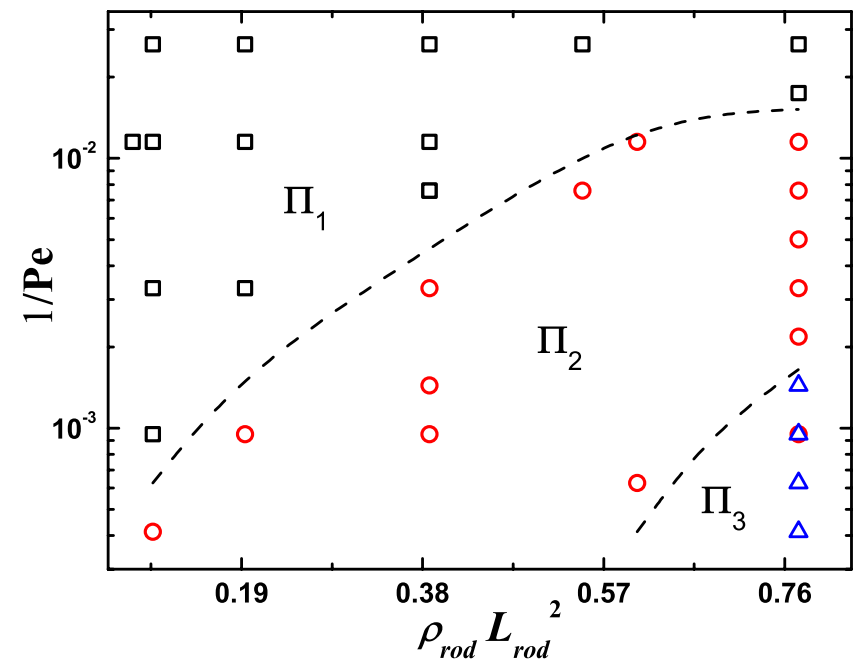

FIG. 5. (Color online) Dynamical phase diagram of swarm behavior. Symbols indicate systems with PDFs $\Pi_{1}$ ( $\square$, black), $\Pi_{2}(\bigcirc$, red), and $\Pi_{3}(\triangle$, blue). All systems were started from a random initial state. The dashed lines are guides to the eye. 


\section{B. Orientational correlation functions}

Although we distinguish three swarming states in our SPR systems, there are only two types of cluster structures. The motile clusters in the $\Pi_{1}$ and $\Pi_{2}$ states consist of polarized rods, as shown in Figs. 2(a) and 2(b). In contrast, the giant clusters found in the $\Pi_{3}$ state consist of a large number of rods blocking each other in their forward motion, as shown in Fig. 2(c).

These two types of clusters can be distinguished by analyzing the orientational correlation function

$$
G(\mathbf{r})=\frac{1}{N_{\text {rod }}\left(N_{\text {rod }}-1\right)} \sum_{i} \sum_{j \neq i}\left\langle\hat{\mathbf{u}}_{i} \cdot \hat{\mathbf{u}}_{j} \cdot \delta\left(\mathbf{r}-\mathbf{r}_{i j}\right)\right\rangle .
$$

Here, $\hat{\mathbf{u}}_{i}$ is the unit vector denoting the orientation of $\operatorname{rod} i$, $\mathbf{r}_{i j}(r, \phi)$ is the vector pointing from the center of mass of rod $i$ to $\operatorname{rod} j$, and $\phi$ is the angle between $\hat{\mathbf{u}}_{i}$ and $\mathbf{r}_{i j} . G(r) \rightarrow 1$ for $r \rightarrow 0$ because two neighboring rods at close distance are always aligned. At large distance, $G(r) \rightarrow 0$.

When the system is in a state characterized by $\Pi_{1}$ or $\Pi_{2}$, $G(\mathbf{r})$ is symmetric with respect to the direction $\phi=0^{\circ}$ with a maximum at $r=0$ [Fig. 6(a)]. The slight elongation of $G(\mathbf{r})$ in the directions $\phi=0^{\circ}$ and $\phi=180^{\circ}$ indicates that the clusters tend to slightly extend in the direction of the average rod orientation due to packaging. The width of $G(\mathbf{r})$ is narrower in the front and wider in the back because of their partially blocked structure [see Fig. 2(d)] and because large clusters are more likely to collide with other clusters head on. If a head-to-head collision does not result in the formation a larger cluster or a blocked structure, the front tips are sharpened due to the "attrition" of the two clusters.

If the system is in the state with a giant cluster, $G(\mathbf{r})$ shows a very different behavior [see Fig. 6(b)]. $G(\mathbf{r})$ still has a positive maximum near $r=0$, which represents a high local orientational order. However, a region with negative correlations, $G<0$, develops, with a minimum at some $\left(r^{\prime}, \phi^{\prime}\right)$. Because all rods point preferentially toward the center of cluster, the propelling forces of the rod nearly compensate each other. Therefore, the locomotion speed of a giant cluster is much smaller than the gliding speed of a single rod. Moreover, the propelling forces generate a net torque due to the deviation of the rod orientations from pointing exactly toward the center of mass, which implies a rotational motion of the giant cluster. $\phi^{\prime}$ is related to this rotation. For $0^{\circ}<\phi^{\prime}$ $<90^{\circ}$, the cluster rotates counterclockwise; for $-90^{\circ}<\phi^{\prime}$ $<0^{\circ}$, it rotates clockwise; and for $\phi^{\prime}=0^{\circ}$, there is no net torque and the giant cluster does not rotate.

\section{Average cluster size}

The average cluster size $\langle n\rangle$ of the system is

$$
\langle n\rangle=\sum_{n} n \Pi(n),
$$

where $\Pi(n)$ is the normalized cluster-size distribution function. $\langle n\rangle$ increases with increasing $\rho_{\text {rod }}$, as shown in Fig. 7(a); in the low-density limit, $\langle n\rangle$ approaches unity. $\langle n\rangle$ decreases with increasing noise level, 1/Pe, as shown in Fig. 7(b). In the $\Pi_{2}$ regime, the system exists in two metastable states,
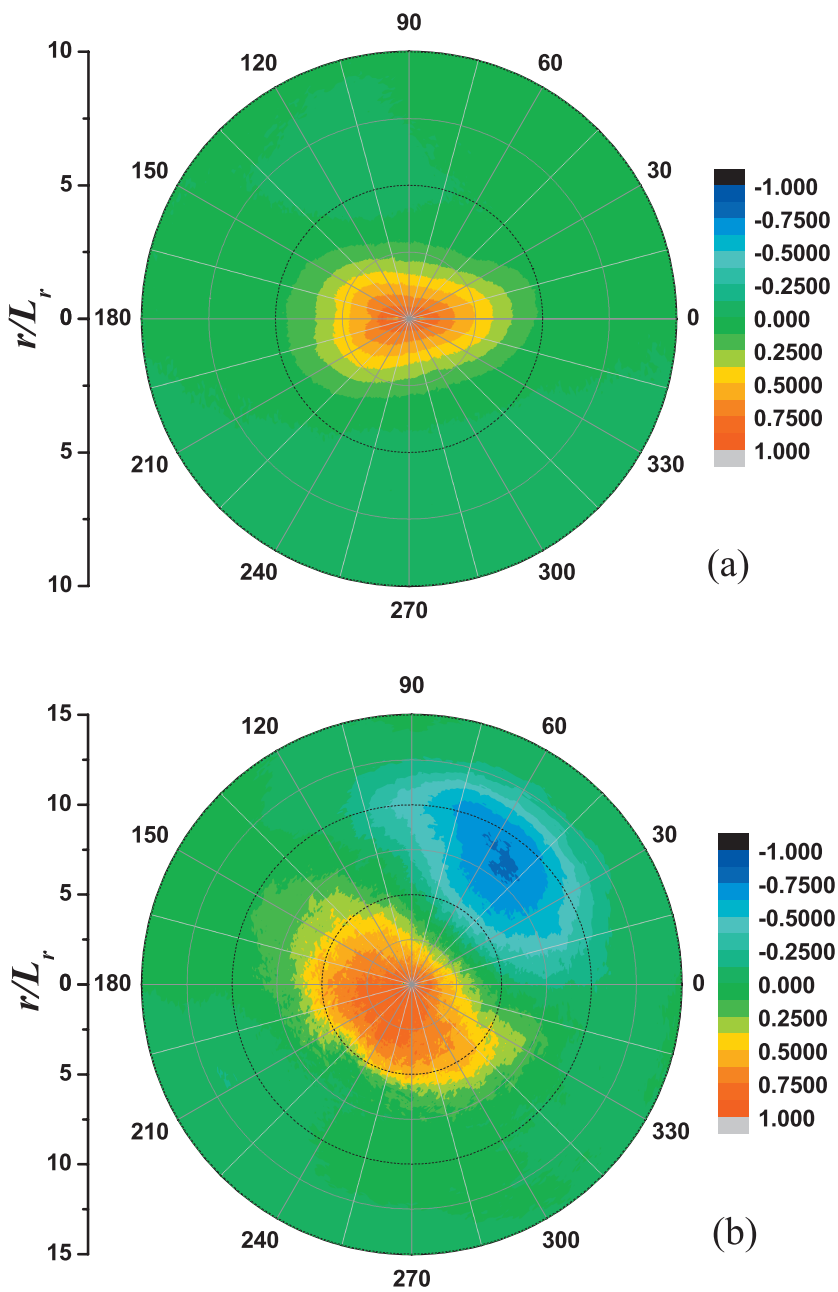

FIG. 6. (Color) The orientational correlation function $G(\mathbf{r})$ as a function of the relative position $\mathbf{r}$ (a) in a system with $\Pi_{2}$ $\left(\rho_{\text {rod }} L_{\text {rod }}^{2}=0.7744\right.$ and $\left.1 / \mathrm{Pe}=0.00501\right)$, where there are motile clusters, and (b) in a system with $\Pi_{3}\left(\rho_{\text {rod }} L_{\text {rod }}^{2}=0.7744\right.$ and $1 / \mathrm{Pe}$ $=0.00041)$ characterized by the presence of a giant cluster.

depending on the initial conditions. With random initial conditions, a "supercooled" state emerges, which transforms into the $\Pi_{3}$ state once a giant-cluster nucleus has formed. This can be seen in Fig. 7(b) for $1 / \mathrm{Pe}=0.000$ 95, where two data points show simulation results with different random numbers for random initial states. With a giant cluster as initial state, the system stays in the $\Pi_{3}$ state unless the noise is large enough to destroy the giant cluster; this occurs in Fig. 7(b) for $1 / \mathrm{Pe}=0.04$. Interestingly, $\langle n\rangle$ shows a power-law decay,

$$
\langle n\rangle \sim \mathrm{Pe}^{\zeta},
$$

in the $\Pi_{1}$ and $\Pi_{2}$ regions when the system starts from a disordered state, with exponent $\zeta \simeq 0.37$.

\section{Cluster lifetime}

We define the lifetime of a cluster as the length of the time during which its members do not change. The lifetime of a cluster is analyzed with a time interval $\Delta \tau=100$; thus, cluster lifetimes less than $\Delta \tau$ cannot be resolved. The aver- 

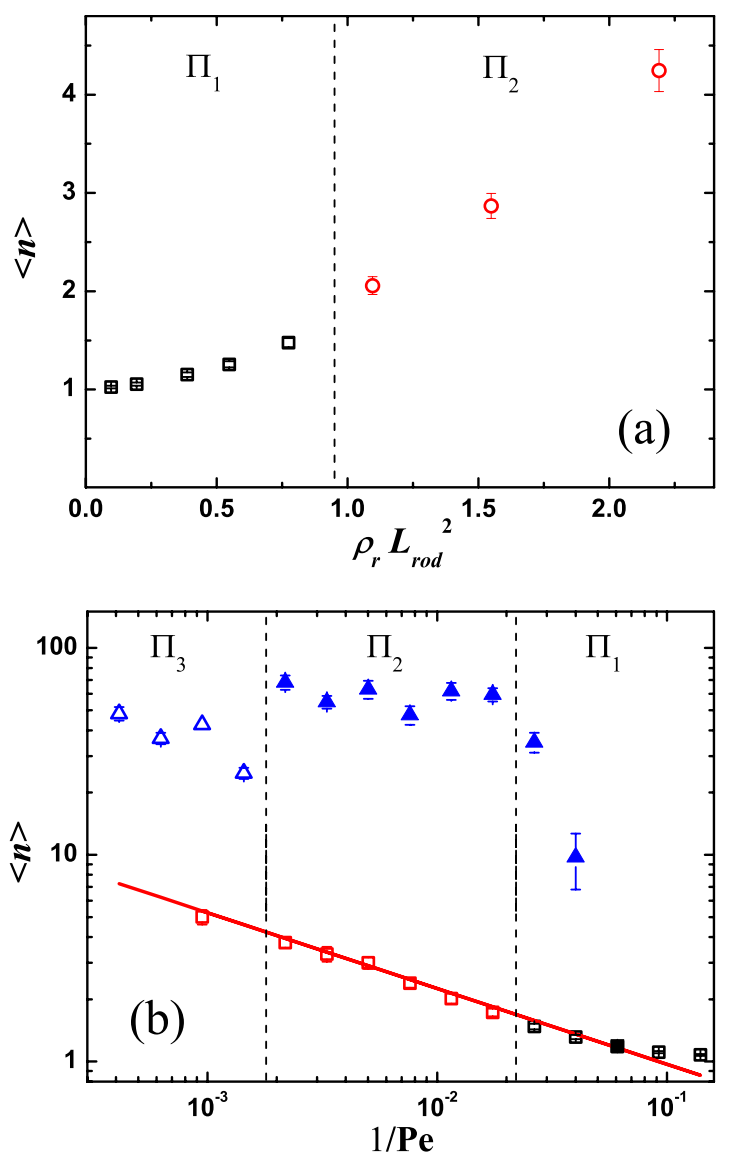

FIG. 7. (Color online) The average cluster size $\langle n\rangle$ as a function of (a) the rod density $\rho_{\text {rod }} L_{\text {rod }}^{2}$ with $1 / \mathrm{Pe}=0.02645$ and (b) the environmental noise $1 / \mathrm{Pe}$ with $\rho_{\text {rod }} L_{\text {rod }}^{2}=0.7744$. The solid (red) line is a fit of the power-law part with exponent $\zeta=0.37$. The dashed (black) lines are the boundaries separating different regions in the phase diagram (Fig. 5). The open symbols represent systems with $\Pi_{3}(\triangle$, blue $), \Pi_{2}(\bigcirc$, red $)$, and $\Pi_{1}(\square$, black), starting from random initial states. The solid symbols represent the systems with $\Pi_{2}(\boldsymbol{\Delta}$, blue) and $\Pi_{1}(\boldsymbol{\square}$, black), starting from a state with a giant cluster.

age cluster lifetime $T_{\text {life }}$ is a function of cluster size $n$.

As shown in Fig. 8, the lifetimes of the clusters of size $n=1$ are always much longer than those of other cluster sizes, because single-rod "clusters" cannot disintegrate. For $n \geq 2, T_{\text {life }}(n)$ decreases smoothly with increasing cluster size. The data for midsize clusters $(2<n<30)$ show an effective power-law dependence,

$$
T_{\text {life }}(n) \sim n^{-\delta},
$$

with an exponent $\delta \simeq 0.2$. Because the environmental noise determines the breakup rate of clusters, $T_{\text {life }}$ increases with decreasing $1 / \mathrm{Pe}$. We only show the lifetime of motile clusters in systems characterized by $\Pi_{1}$ and $\Pi_{2}$. The giant clusters found in the state characterized by $\Pi_{3}$ can persist for a very long time until a sufficiently large fluctuation occurs.

To understand the dependence of the cluster lifetime on $n$, we can assume that only single rods are lost at the cluster surface [37]. In this case, the probability to loose a rod per unit time is proportional to the perimeter length, which

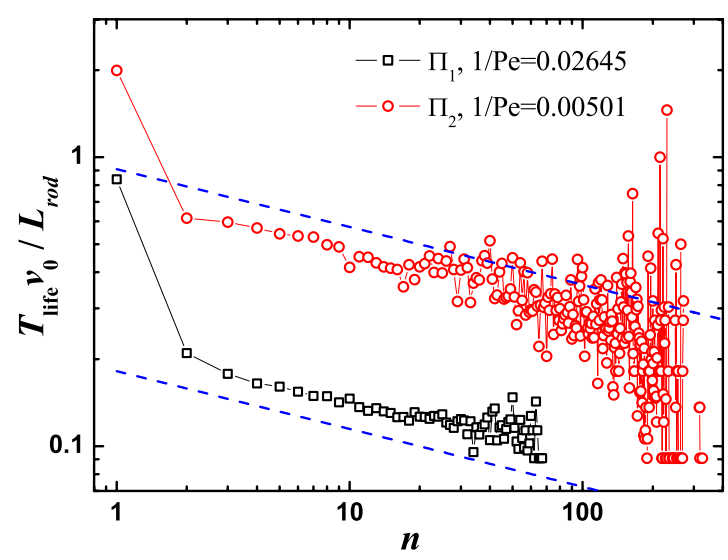

FIG. 8. (Color online) Average cluster lifetime $T_{\text {life }}(n)$ for systems with the same rod number density $\rho_{\text {rod }} L_{\text {rod }}^{2}=0.7744$ but with a different noise level, as indicated. The dashed lines are power laws (21) with an exponent $\delta=0.2$.

scales as $n^{1 / 2}$ (for compact clusters of approximately circular shape). Therefore, this simple argument implies a scaling law (21) with exponent $\delta=0.5$. The growth of clusters is more complex since it can occur by collision with all types of other clusters; however, the collision cross section should again be proportional to $n^{1 / 2}$. The value of $\delta=0.5$ is considerably smaller (corresponding to shorter lifetimes for larger clusters) than that observed in our simulations. This indicates that there must be another mechanism of cluster decay. Indeed, the typical cluster configurations of Fig. 2(d) indicate that only at few places along the perimeter, rods may have the possibility to leave the cluster.

\section{E. Finite-size effects}

In our simulations, the finite simulation-box size implies a finite number of particles. A cluster can never grow larger than the total number of rods in the system. Consequently, all quantities related to the cluster size, such as the cluster-size distribution $\Pi$ and the stationary average cluster size $\langle n\rangle$, display finite-size effects. Similarly, density fluctuations at the scale of the simulation-box size are suppressed.

For the probability density function $\Pi(n)$, the absence of cluster larger than $N_{\text {rod }}$ does not only introduce a cutoff at large cluster size, but also affects the exponent $\beta$ of the power-law part, as shown in Fig. 9. For systems with $\Pi_{1}$, the data for small box sizes $\left(L_{x}=L_{y}=4.5 L_{\text {rod }}\right.$ and $\left.13 L_{\text {rod }}\right)$ still obey a power-law decay at small $n$, without an obvious change in the exponent, as shown in the inset of Fig. 9, but they deviate from the power law when $n$ approaches $N_{\text {rod }}$. When the simulation box is large $\left(L_{x}=L_{y}=36 L_{\text {rod }}\right.$ and $51 L_{\text {rod }}$ ), the PDFs almost coincide, and their exponential cutoffs are observed at the same value of $n$; also, $\beta$ approaches an asymptotic value when $L_{x}$ increases. Therefore, we conclude that our results for the larger systems represent the thermodynamic limit. Similarly, the power-law part of $\Pi_{2}$ extends with increasing box size, and the location of the prominent shoulder shifts to larger cluster size. The finitesize effects are significantly stronger for systems in the $\Pi_{3}$ region of the phase diagram. When the system is too small, 


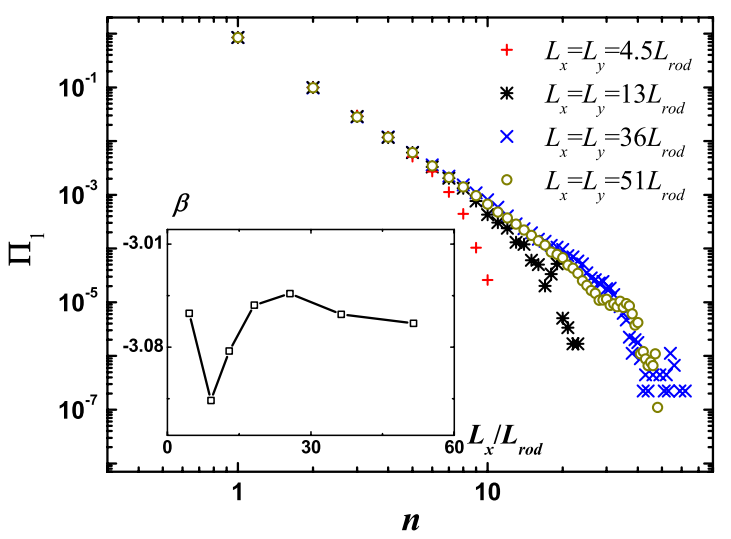

FIG. 9. (Color online) Effect of finite system size on the probability density distribution function $\Pi_{1}(n)$ for $\rho_{\text {rod }} L_{\text {rod }}^{2}=0.7744$, $1 / \mathrm{Pe}=0.04009$, and different simulation-box sizes, as indicated. The inset shows the exponent $\beta$ of the power-law part of $\Pi_{1}(n)$ as a function of the size $L_{x}$ of the simulation box.

the total rod number is not sufficient to trigger the formation of a blocked structure. The system then stays in a $\Pi_{2}$ state. This supports the claim that the state with $\Pi_{2}$ is a supercooled state. We believe that the absence of the $\Pi_{3}$ state in Ref. [37] is due to finite-size effect; a system of only 100 rods is too small to form a blocked structure.

The dependence of the average cluster size $\langle n\rangle$ on the linear system size $L_{x}$ is shown in Fig. 10. For systems with $\Pi_{1}$ and $\Pi_{2},\langle n\rangle$ increases with $L_{x}$ and eventually reaches a plateau value. For the system with $\Pi_{3},\langle n\rangle$ strongly diverges when $L_{x}$ increases. Thus, $\langle n\rangle$ can be considered as an intensive quantity in the first two states and as an extensive quantity in the third state.

Suppose that the probability density function $\Pi(n)$ obeys a power law for all cluster sizes,

$$
\Pi(n)=\frac{1+\beta}{N^{1+\beta}-1} n^{\beta},
$$

where $\beta<-1$ and $N=\rho_{\text {rod }} L_{x} L_{y}$ is the total number of rods in the system. It is easy to verify that for $N \gg 1$, where sums

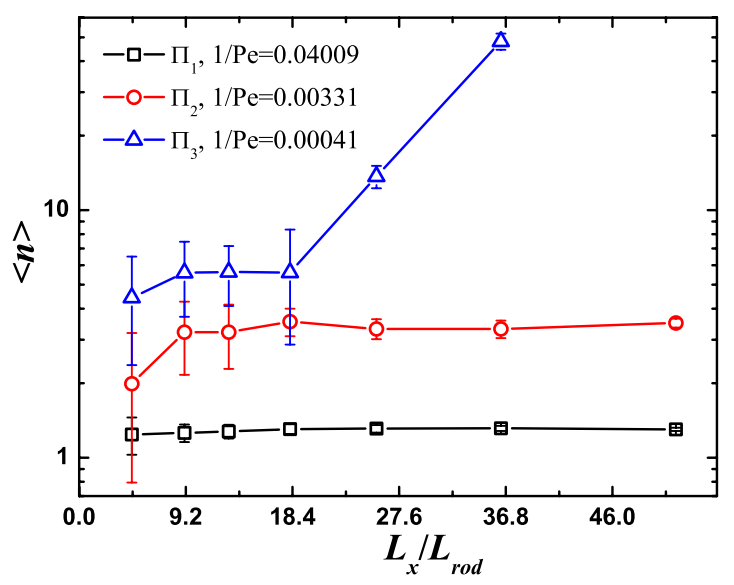

FIG. 10. (Color online) The average cluster size $\langle n\rangle$ as a function of the size $L_{x}$ of the simulation box for the three clustering states. The number density is $\rho_{\text {rod }} L_{\text {rod }}^{2}=0.7744$ in all systems.

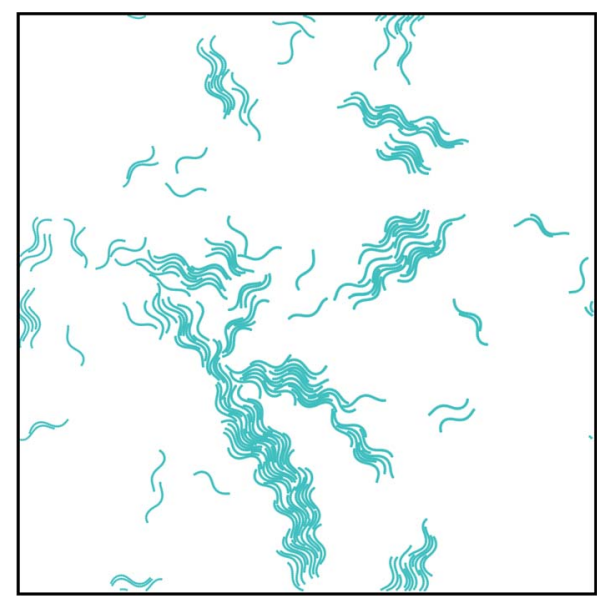

FIG. 11. (Color online) Snapshot of a multiflagellum system in a MPC fluid. The parameters are $\rho_{f l} L_{f l}^{2}=1.5625$ and $\sigma_{f l}=0.1 \%$. The black box shows the boundary of the simulation box. Periodic boundary conditions are employed. For a movie see Ref. [53].

over $n$ can be well approximated by integrals, $\int_{1}^{N} \Pi(n) d n=1$, so that $\Pi(n)$ is properly normalized. The sharp drop due to the limited box size is neglected. In this case, the average cluster size of the system is obtained to be

$$
\langle n\rangle= \begin{cases}-(1+\beta) N^{2+\beta} /(2+\beta), & -2<\beta<-1 \\ \left(1-N^{-1}\right)^{-1} \ln N, & \beta=-2 \\ (1+\beta) /(2+\beta), & \beta<-2 .\end{cases}
$$

For $-2<\beta<-1$, the average cluster size strongly depends on the total number $N$ of rods, whereas for $\beta<-2,\langle n\rangle$ is independent of $N$. For large negative $\beta,\langle n\rangle$ approaches unity, which means that all rods are gliding freely.

In our simulations, the effective exponents in the $\Pi_{1}$ and $\Pi_{2}$ regimes are $-6 \lesssim \beta \lesssim-2.5$ and $-2.5 \lesssim \beta \lesssim-2.0$, respectively (see Fig. 4). Thus, Eq. (23) implies that finite-size effects are weak in the $\Pi_{1}$ regime and are pronounced in the $\Pi_{2}$ regime, in agreement with the simulation results of Fig. 10. [Equation (23) does not apply to the $\Pi_{3}$ state since the assumption of a power-law dependence (22) does not hold.]

\section{SWARMING BEHAVIOR OF FLAGELLA IN A MPC FLUID}

Multiflagellum systems show a similar swarming behavior, consisting of aggregation and clustering, as observed in Sec. III for self-propelled rods (see Fig. 11 and movie [53]). Synchronization of the flagellar beat and attraction and alignment of flagella do not only arise from volume exclusion, as in the SPR systems, but are also triggered by the hydrodynamic interactions between the sinusoidally undulating bodies $[43,44]$. At the same time, hydrodynamic interactions between flagella act as a source of environmental noise, which causes the flagella trajectories to fluctuate strongly.

\section{A. Hydrodynamic synchronization, attraction, and aggregation}

The synchronization and attraction of two flagella are shown in Fig. 12. Synchronization is achieved within about 


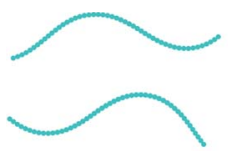

(a)

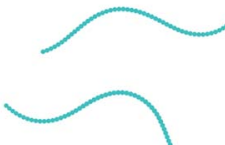

(b)

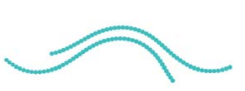

(c)
FIG. 12. (Color online) Synchronization and attraction of two flagella. The flagella have the same beating frequency $f=1 / 120$, and a phase difference $\Delta \varphi=0.5 \pi$. The snapshots are taken at the times (a) $t f=0.167$, (b) $t f=4.17$, and (c) $t f=22.2$.

four beats, while the formation of a tight pair from an initial distance of about one third of the flagellar length takes about 20 beats. The flow field of a flagellum is shown in Fig. 13. The flow field at a certain time in the beating cycle [Fig. 13(a)] shows the formation of two vortices, which propagate from the front to the rear end as the flagellum moves forward.

The hydrodynamic interaction of swimmers depends on the type of self-propulsion. The average flow field of flagellum, integrated over the whole beating cycle, demonstrates that the flagellum, which might be expected to be a "neutral" swimmer (i.e., neither a pusher nor a puller), is indeed a very weak pusher-where the dominant propulsion is located closer to the rear end-because the line connecting the centers of the two vortices intersects the average flagellum shape
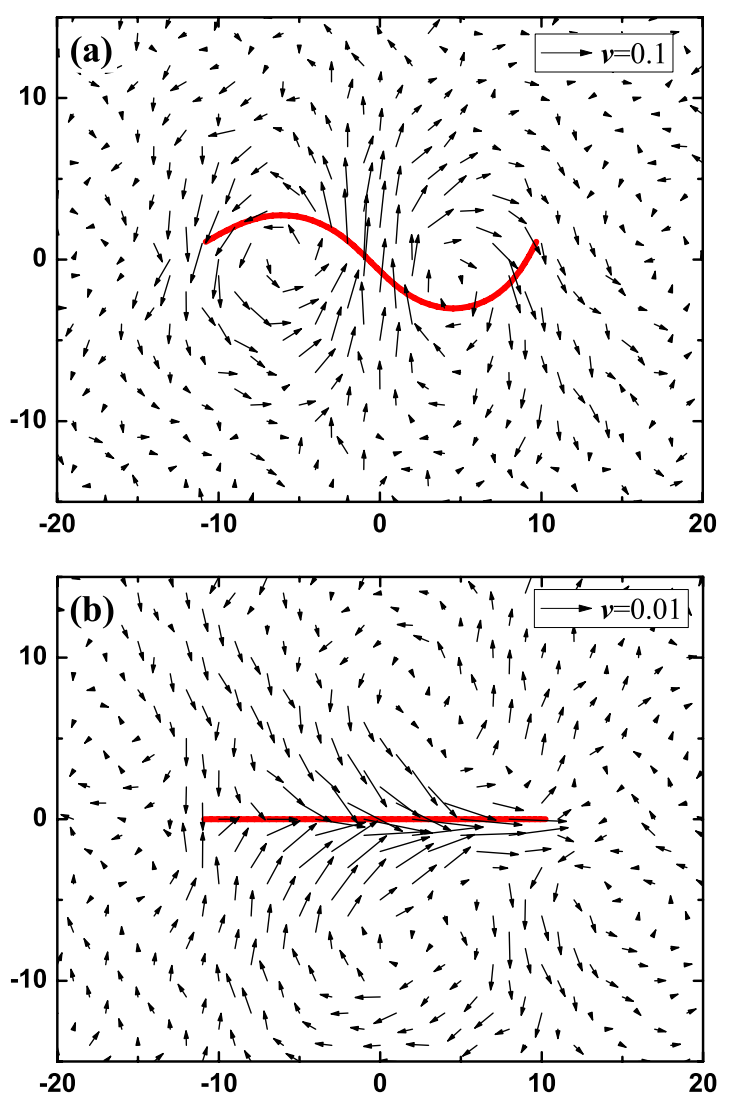

FIG. 13. (Color online) Flow field of a single flagellum (a) at a fixed time in the beating cycle and (b) averaged over the whole beating cycle. A snapshot of the flagellum and the average flagellum shape are superimposed in (a) and (b), respectively. The scale bar indicates the magnitude of the flow velocities.

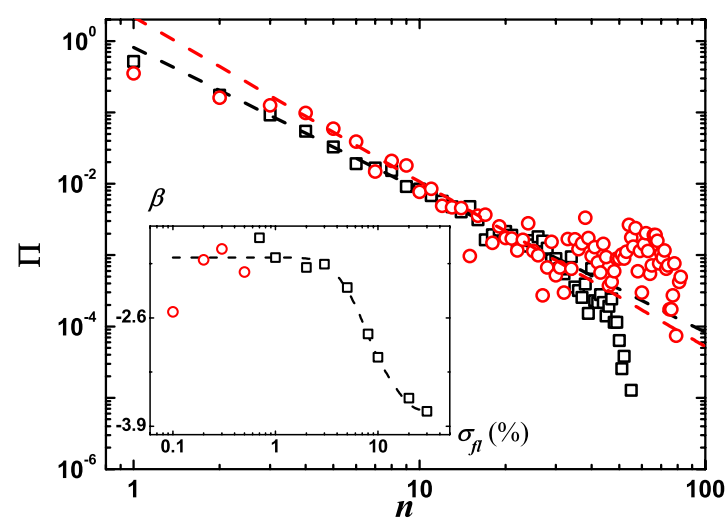

FIG. 14. (Color online) The two different types of cluster-size probability density functions $\Pi_{1}(n)$ with $\sigma_{f l}=2 \%$ ( $\square$, black) and $\Pi_{2}(n)$ with $\sigma_{f l}=0.1 \%(\bigcirc$, red), observed in multiflagellum systems with density $\rho_{f l} L_{f l}^{2}=1.5625$. The dashed lines are fits to the powerlaw parts of each PDF. The inset shows the exponent $\beta$ as a function of the variance $\sigma_{f l}$ of the frequency distribution. Symbols indicate the systems characterized by $\Pi_{2}\left(\bigcirc\right.$, red) and $\Pi_{1}(\square$, black). The dashed line is a guide to the eye.

behind its midpoint [Fig. 13(b)]. This generates an inflow from both sides of the flagellum near the front end, which is responsible for hydrodynamic attraction [40,41].

In multiflagellum systems, large clusters can form by collisions of smaller clusters, supported by the hydrodynamic attraction between neighboring flagella; large clusters can disintegrate into smaller components due to the diversity of flagellar frequencies or the hydrodynamic flow fields of other clusters. With hydrodynamic interactions, large clusters of flagella are usually strongly extended in their directions of motion, as shown in Fig. 11 and movie [53]. The flagella inside the cluster are well synchronized. This structure is reminiscent of the "sperm-train" structure observed in rodent-sperm experiments [27,28]. The elongated clusters can extend to distances as large as the side length of the simulation box, which induces strong finite-size effects.

Similar to the definition of a rod cluster in Sec. III, a flagellum cluster is defined as a set of flagella that are connected or neighbors either directly or through other agents at a given moment in time. Its size is the number $n$ of flagella it contains. A freely swimming single flagellum is considered as a cluster of size $n=1$.

\section{B. Cluster-size distributions}

Both probability density functions $\Pi_{1}$ and $\Pi_{2}$ are observed in our multiflagellum systems, as shown in Fig. 14. The variance $\sigma_{f l}$ of the distribution of beat frequencies is used as a measure of the noise level. At low $\rho_{f l}$ or high $\sigma_{f l}$, we find $\Pi_{1}$; at high $\rho_{f l}$ or low $\sigma_{f l}$, we observe $\Pi_{2}$. In contrast to $\Pi_{2}$ for SPR systems in Sec. III A, $\Pi_{2}$ for flagella systems displays a deviation from the power-law behavior for very small cluster sizes $n=1$ and $n=2$. We believe that this is due to the hydrodynamic synchronization and attraction of neighboring flagella. For flagella, we have never observed a giant cluster with a blocked structure, in contrast to the SPR system of Fig. 2(c). 


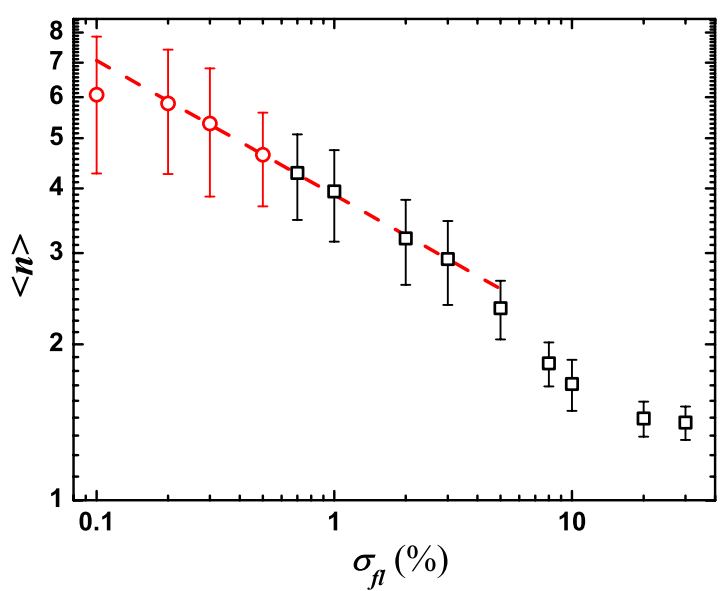

FIG. 15. (Color online) Stationary average cluster size $\langle n\rangle$ versus the variance $\sigma_{f l}$ of the frequency distribution, for flagellar density $\rho_{f l} L_{f l}^{2}=1.5625$. Symbols indicate systems with $\Pi_{1}$ ( $\square$, black) and $\Pi_{2}(\bigcirc$, red). The error bars are the standard deviation of the fluctuations. The dashed line indicates a power-law decay with an exponent $\zeta=0.26$.

Although the distribution of beating frequencies is an internal property of the swimmers, the influence of $\sigma_{f l}$ on the exponent $\beta$ of Eq. (17) is similar to the influence of the environmental noise in our previous SPR simulations, as shown in the inset of Fig. 14. $\beta$ is nearly constant for $\sigma_{f l}$ $<3 \%$, and then decreases smoothly with increasing $\sigma_{f l}$.

The average cluster size $\langle n\rangle$ in the stationary state is a function of $\sigma_{f l}$, as shown in Fig. 15. Increasing $\sigma_{f l}$ results in an increase of the overall breakup rate; hence, $\langle n\rangle$ decreases. In the large- $\sigma_{f l}$ limit, $\langle n\rangle$ approaches unity, corresponding to a disordered state with randomly distributed flagella. The power-law decay

$$
\langle n\rangle \sim \sigma_{f l}^{-\zeta}
$$

of the average cluster size with exponent $\zeta \simeq 0.26$ emphasizes the universality of the swarming behavior of rSPP systems in two dimensions. The power-law scaling of $\langle n\rangle$ as a function of $\sigma_{f l}$ implies a divergence when $\sigma_{f l} \rightarrow 0$. We believe that the small deviation from the power-law behavior for $\sigma_{f l}=0.1 \%$ in Fig. 15, as well as the deviation of $\beta$ from the plateau value for $\sigma_{f l}=0.1 \%$ in Fig. 14 , is due to finitesize effects.

\section{Cluster lifetimes}

The average cluster lifetime $T_{\text {life }}(n)$ decreases as an effective power-law function of cluster size $n$ [see Eq. (21)] with an exponent $\delta \simeq 0.5$, as shown in Fig. 16. The value of $\delta$ is very close to the prediction based on the assumption of a mechanism of particle accumulation and shedding proportional to the cluster perimeter, as presented in Sec. III D. This good agreement provides further evidence for the different mechanisms of cluster stabilization for rods and flagella, which are a (partially) blocked motion and a hydrodynamic attraction, respectively.

Note that the system size of the flagella simulations is not as large as for the SPR systems. Thus, the effective power

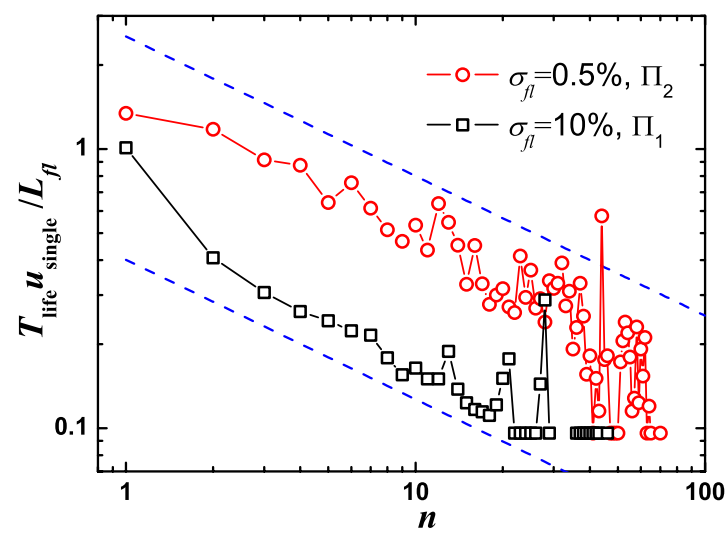

FIG. 16. (Color online) The lifetime $T_{\text {life }} v_{\text {single }} / L_{f l}$ of flagella clusters versus their size $n$. The flagella number density is $\rho_{f l} L_{f l}^{2}$ $=1.5625$. The dashed lines indicate a power law with exponent $\delta$ $=0.5$.

law can only be observed over a smaller range of cluster sizes. In SPR simulations, single rods $(n=1)$ always have a much longer lifetime compared to expectation from the effective power law (see Fig. 8). In contrast, for flagella with full hydrodynamic interactions, $T_{\text {life }}(1)$ is much closer to the power-law extrapolation and can even be lower than the power-law prediction (e.g., for $\sigma_{f l}=0.5 \%$ in Fig. 16).

\section{Comparison of sperm and flagella}

As explained in Sec. II B, our model of a flagellum differs from the model of a sperm employed in Ref. [43] by the absence of a passive midpiece and a circular head. Also, in the sperm simulations [43], two sine waves were present on the tail, while a single sine wave is present on the flagellum.

How similar or different is the collective behavior of sperm and flagella? There are three different aspects to this question. Synchronization depends mainly on the interaction of the time-dependent oscillatory flow field of two neighboring flagella $[24,44]$ and is therefore very similar, as can be seen from the results presented in Sec. IV A and those of Ref. [43]. On the other hand, the hydrodynamic attraction of sperm and flagella is quite different. A sperm cell, consisting of a flagellum and a large head, is clearly a pusher, as demonstrated by the average flow field of a sperm in Fig. 17. The flagellum pushes the fluid backward in both cases, but the bulky head of the sperm drags the fluid forward much more strongly, which generates the characteristic sidewise inflow of fluid toward the midpiece region $[40,41,45]$. In contrast, flagella are very weak pushers, as demonstrated in Fig. 13(b) above. Therefore, sperm have a stronger hydrodynamic attraction than flagella.

Finally, the swarming behavior in both flagella and sperm system is characterized by cluster-size distributions and the dependence of the average cluster size on the width $\sigma_{f l}$ of the distribution of beat frequencies. While the cluster-size distribution of flagella follows a power-law decay over a wide range, it was not possible to clearly identify a power-law behavior for sperm in Ref. [43] due to the relatively small systems of 25 and 50 sperm. The average cluster size is 


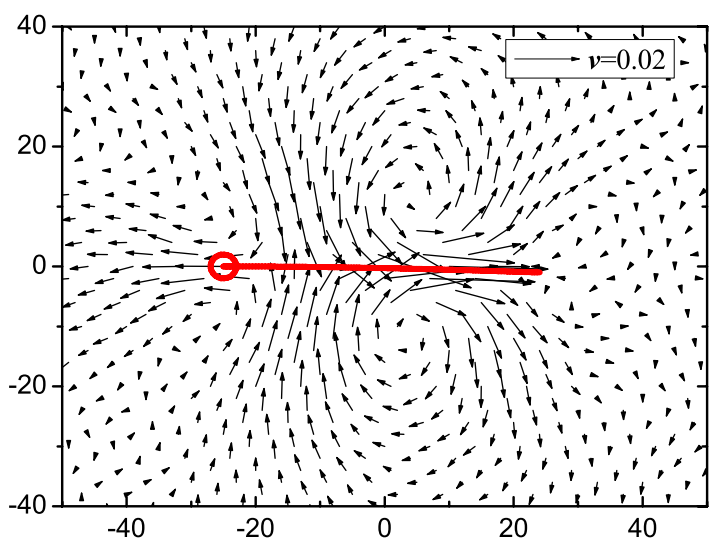

FIG. 17. (Color online) Flow field of a single sperm, beating with two sine waves on its tail, averaged over the whole beating cycle. The average sperm shape is superimposed. The scale bar indicates the magnitude of the flow velocities.

found to depend on $\sigma_{f l}$ as $\langle n\rangle \sim \sigma_{f l}^{-\zeta}$, with $\zeta=0.20$ for sperm [43] and $\zeta=0.26$ for flagella. Larger systems have to be investigated to see whether the exponents $\zeta$ for sperm and flagella are different or not. In any case, the stronger hydrodynamic attraction of sperm, which favors larger cluster sizes, is partially offset by the bulky head of sperm, which implies that the sperm clusters in Ref. [43] are more loosely packed than the flagella clusters studied here.

\section{SUMMARY AND CONCLUSIONS}

We have simulated systems of rigid rods propelled by a constant force along their long axis and systems of flagella propelled by a sinusoidal beating motion in two dimensions. In both systems, we observe cluster formation and breakup, controlled by the particle density and the internal or external noise. In our simulations, the particle density is always much lower than the critical density of a nematic phase in thermal equilibrium.

Without any attractive potential, self-propelled rods (SPRs) exhibit an aggregation behavior triggered only by volume exclusion. Three characteristic types of cluster-size probability density functions $\Pi(n)$ appear in different regions of a dynamic phase diagram of stationary states. At high noise and low density, the system is characterized by $\Pi_{1}$, which shows a power-law distribution over a range of cluster sizes, with an exponential cutoff at large cluster sizes. At low noise and high density, the system is in a state characterized by $\Pi_{3}$, which has a peak at sizes near the total number of particles in the system, representing a giant cluster. Systems in an intermediate region of noise and density are characterized by $\Pi_{2}$, which is a transition state between $\Pi_{1}$ and $\Pi_{3}$. It has a bimodal shape, with a power-law decay at small cluster sizes and a shoulder at larger sizes. Clusters in $\Pi_{1}$ and $\Pi_{2}$ systems retain a high motility, whereas the giant clusters found in the third state are almost immobile due to their blocked configuration. The average cluster size at equilib- rium, directly related to cluster-size distribution $\Pi$, displays a power-law dependence with decreasing noise amplitude before the system reaches the $\Pi_{3}$ state.

Sinusoidally beating flagella were simulated in a lowReynolds-number fluid with full hydrodynamics as an example of self-propelled rodlike particles with explicit propulsion mechanism. Flagella synchronize their beats and attract each other through the hydrodynamic interactions. Despite the different propulsion mechanisms, the basic swarm behavior of aggregation and clustering observed for swimming flagella is remarkably similar to the behavior seen in SPR systems. We observe both $\Pi_{1}$ and $\Pi_{2}$ cluster-size probability density functions by varying the width $\sigma_{f l}$ of the flagellar beat-frequency distribution, which acts as a source of internal noise in the system. The average cluster size also displays a power-law dependence on $\sigma_{f l}$, as for SPR systems.

Despite these similarities in the clustering behavior, the two systems show some important differences. They can be traced back to the hydrodynamic attraction between beating flagella, which is absent in our simulations of self-propelled rods. First, the configurations of the flagella clusters consist of tightly stacked flagella with synchronized shapes and extend in their moving directions. Those elongated clusters are reminiscent of the huge mobile sperm trains observed in rodent-sperm experiments [27]. Clusters in the SPR systems are more compact and have a wedgelike structure, which arises from the partially blocked rod motion responsible for the cluster aggregation, as well as from collisions with other clusters. Second, the $\Pi_{3}$ state of a completely blocked structure, which is observed for SPR at high density and low noise, does not seem to exist in flagellar systems. Third, the cluster lifetimes decay with different effective power laws: $\delta=0.2$ for SPR and $\delta=0.5$ for flagella. Finally, hydrodynamic interactions between different flagella clusters act as an additional source of noise and contribute to increase the breakup rate.

The existence of the giant immobile cluster should depend sensitively on the aspect ratio and the type and range of the interactions between self-propelled rods, where longer rods and shorter-range interaction favors the giant-cluster formation. This conclusion follows from the result of Ref. [37] for rods of aspect ratio $L_{\text {rod }} / l_{b} \leq 12$ that $\Pi_{1}-\Pi_{2}$ boundary shifts to higher density with decreasing rod length, and from our result in Fig. 7 that the $\Pi_{2}$ state corresponds to supercooled liquid state which transforms into the $\Pi_{3}$ state once a giantcluster nucleus has formed. Blocked clusters were not seen in Ref. [37] for rod lengths $L_{\text {rod }} / l_{b} \leq 12$ due to the relatively small system size with $N_{\text {rod }}=100$. However, blocked states were observed in Ref. [36] for a much larger rod length, $L_{\text {rod }} / l_{b}=40$, already for a system of only about 50 rods at density $\rho_{\text {rod }} L_{\text {rod }}^{2}=2$.

Our simulations have been restricted to the isotropic phase of rods in thermal equilibrium. It will be interesting to see in the future whether immobile blocked states can also exist (or even dominate) in the nematic regime or whether they are suppressed by the preferred rod orientation. In light of our results, we conclude that different systems of rodlike self-propelled particles display not only a universal swarming behavior, but also specific properties related to their propulsion mechanisms and the presence or absence of hydrodynamic interactions. 


\section{ACKNOWLEDGMENTS}

We thank Jens Elgeti and Roland Winkler for stimulating discussions. Y.Y. acknowledges support by the International Helmholtz Research School on Biophysics and Soft Matter
(IHRS BioSoft). V.M. is grateful to the RISE program of the DAAD (Germany) and to NSERC (Canada) for financial support. This work was supported in part by the VW foundation through the program "Computational Soft Matter and Biophysics."
[1] T. Vicsek, A. Czirók, E. Ben-Jacob, I. Cohen, and O. Shochet, Phys. Rev. Lett. 75, 1226 (1995).

[2] J. Toner and Y. Tu, Phys. Rev. Lett. 75, 4326 (1995).

[3] R. A. Simha and S. Ramaswamy, Phys. Rev. Lett. 89, 058101 (2002).

[4] S. Ramaswamy, R. A. Simha, and J. Toner, EPL 62, 196 (2003).

[5] F. Peruani, A. Deutsch, and M. Bär, Eur. Phys. J. Spec. Top. 157, 111 (2008).

[6] A. Baskaran and M. C. Marchetti, Phys. Rev. Lett. 101, 268101 (2008).

[7] A. Baskaran and M. C. Marchetti, Phys. Rev. E 77, 011920 (2008).

[8] E. Bertin, M. Droz, and G. Grégoire, J. Phys. A: Math. Theor. 42, 445001 (2009).

[9] B. Szabó, G. J. Szöllösi, B. Gönci, Z. Jurányi, D. Selmeczi, and T. Vicsek, Phys. Rev. E 74, 061908 (2006).

[10] G. Grégoire and H. Chaté, Phys. Rev. Lett. 92, 025702 (2004).

[11] C. Huepe and M. Aldana, Phys. Rev. Lett. 92, 168701 (2004).

[12] M. R. D’Orsogna, Y. L. Chuang, A. L. Bertozzi, and L. S. Chayes, Phys. Rev. Lett. 96, 104302 (2006).

[13] M. Aldana, V. Dossetti, C. Huepe, V. M. Kenkre, and H. Larralde, Phys. Rev. Lett. 98, 095702 (2007).

[14] H. Chaté, F. Ginelli, G. Grégoire, and F. Raynaud, Phys. Rev. E 77, 046113 (2008).

[15] F. Ginelli, F. Peruani, M. Bär, and H. Chaté, Phys. Rev. Lett. 104, 184502 (2010).

[16] C. Huepe and M. Aldana, Physica A 387, 2809 (2008).

[17] X.-L. Wu and A. Libchaber, Phys. Rev. Lett. 84, 3017 (2000); 86, 557 (2001); G. Grégoire, H. Chaté, and Y.-H. Tu, ibid. 86, 556 (2001).

[18] C. Dombrowski, L. Cisneros, S. Chatkaew, R. E. Goldstein, and J. O. Kessler, Phys. Rev. Lett. 93, 098103 (2004).

[19] A. Sokolov, I. S. Aranson, J. O. Kessler, and R. E. Goldstein, Phys. Rev. Lett. 98, 158102 (2007).

[20] E. Ben-Jacob, I. Cohen, and H. Levine, Adv. Phys. 49, 395 (2000).

[21] J. M. Kuner and D. Kaiser, J. Bacteriol. 151, 458 (1982).

[22] O. A. Igoshin, R. Welch, D. Kaiser, and G. Oster, Proc. Natl. Acad. Sci. U.S.A. 101, 4256 (2004).

[23] M. S. Alber, M. A. Kiskowski, and Y. Jiang, Phys. Rev. Lett. 93, 068102 (2004).

[24] G. Taylor, Proc. R. Soc. London, Ser. A 209, 447 (1951).

[25] J. Gray, J. Exp. Biol. 35, 96 (1958).

[26] J. Gray and H. W. Lissmann, J. Exp. Biol. 41, 135 (1964).

[27] H. D. M. Moore, K. Dvořáková, N. Jenkins, and W. G. Breed,
Nature (London) 418, 174 (2002)

[28] S. Immler, H. D. M. Moore, W. G. Breed, and T. R. Birkhead, PLoS ONE 2, e170 (2007).

[29] I. H. Riedel, K. Kruse, and J. Howard, Science 309, 300 (2005).

[30] W. F. Paxton et al., J. Am. Chem. Soc. 126, 13424 (2004).

[31] Y. Hong, N. M. K. Blackman, N. D. Kopp, A. Sen, and D. Velegol, Phys. Rev. Lett. 99, 178103 (2007).

[32] V. Narayan, S. Ramaswamy, and N. Menon, Science 317, 105 (2007).

[33] A. Kudrolli, G. Lumay, D. Volfson, and L. S. Tsimring, Phys. Rev. Lett. 100, 058001 (2008).

[34] L. Rothschild, Nature (London) 198, 1221 (1963).

[35] R. F. Kayser and H. J. Raveché, Phys. Rev. A 17, 2067 (1978).

[36] P. Kraikivski, R. Lipowsky, and J. Kierfeld, Phys. Rev. Lett. 96, 258103 (2006).

[37] F. Peruani, A. Deutsch, and M. Bär, Phys. Rev. E 74, 030904(R) (2006).

[38] J. Elgeti and G. Gompper, EPL 85, 38002 (2009).

[39] H. H. Wensink and H. Löwen, Phys. Rev. E 78, 031409 (2008).

[40] E. Lauga and T. R. Powers, Rep. Prog. Phys. 72, 096601 (2009).

[41] T. Ishikawa, J. R. Soc., Interface 6, 815 (2009).

[42] D. Saintillan and M. J. Shelley, Phys. Rev. Lett. 99, 058102 (2007).

[43] Y. Yang, J. Elgeti, and G. Gompper, Phys. Rev. E 78, 061903 (2008).

[44] G. J. Elfring and E. Lauga, Phys. Rev. Lett. 103, 088101 (2009).

[45] J. Elgeti, U. B. Kaupp, and G. Gompper, Biophys. J. 99, 1018 (2010).

[46] R. Kapral, Adv. Chem. Phys. 140, 89 (2008).

[47] G. Gompper, T. Ihle, D. M. Kroll, and R. G. Winkler, Adv. Polym. Sci. 221, 1 (2009).

[48] M. Ripoll, K. Mussawisade, R. G. Winkler, and G. Gompper, EPL 68, 106 (2004).

[49] I. H. Riedel-Kruse, A. Hilfinger, J. Howard, and F. Jülicher, HFSP J. 1, 192 (2007).

[50] T. Ihle and D. M. Kroll, Phys. Rev. E 63, 020201(R) (2001).

[51] N. Kikuchi, C. M. Pooley, J. F. Ryder, and J. M. Yeomans, J. Chem. Phys. 119, 6388 (2003).

[52] T. Ihle and D. M. Kroll, Phys. Rev. E 67, 066706 (2003).

[53] See supplementary material at http://link.aps.org/supplemental/ 10.1103/PhysRevE.82.031904 for movies of the clustering and aggregation of self-propelled rods and swimming flagella. 